\title{
SPINORS AND CANONICAL HERMITIAN FORMS
}

\author{
by P. L. ROBINSON
}

(Received 23 February, 1987)

Introduction. The space $S$ of spinors associated to a $2 m$-dimensional real inner product space $(V, B)$ carries a canonical Hermitian form \langle\rangle determined uniquely up to real multiples. This form arises as follows: the complex Clifford algebra $C(V)$ of $(V, B)$ is naturally provided with an antilinear involution; this induces an involution on End $S$ via the spin representation; this is the adjoint operation corresponding to \langle\rangle .

Suppose that $B$ has signature $p-q$ and rank $p+q=2 m$. If $p$ and $q$ are odd, then \langle\rangle is zero on each half-spin space; if $p$ and $q$ are even, then the half-spin spaces are mutually orthogonal under \langle\rangle . The signature of \langle\rangle depends rather severely on the type $(p, q)$ of $B$. From above, if $p$ and $q$ are odd then \langle\rangle has zero signature. If $p$ and $q$ are even and non-zero then \langle\rangle has zero signature on each half-spin space. If $B$ is definite then \langle\rangle is definite on each half-spin space: of like signs when $B$ is negative, of opposite signs when $B$ is positive.

A few remarks are in order. $C(V)$ actually admits two distinguished involutions, related by the grading automorphism: we select one of these; choosing the other engenders a parallel theory, with $B$ and $-B$ interchanged. We restrict our attention to even dimensions since in odd dimensions the Clifford algebra is not a full matrix algebra. We deal exclusively with real and complex scalars, though some of our results and proofs are valid over more general fields with involution. Elementary modifications of the methods adopted here lead rapidly to the existence and properties of the familiar canonical bilinear forms on spaces of spinors.

In Section 1 we present a detailed account of the canonical Hermitian forms on spin spaces. In Section 2 we present some simple examples; in particular, we discuss the twistor space associated with Minkowski space. Throughout, we assume a certain familiarity with the structure of Clifford algebras: see any of the references listed at the end of this paper.

1. The Hermitian forms. Let $V$ be a $2 m$-dimensional real vector space endowed with an inner product $B$ of type $(p, q)$; thus, $B$ has rank $p+q=2 m$ and signature $p-q$. The dimension of $V$ being even, $p$ and $q$ have like parity; we shall refer to $B$ as even or odd according to whether this parity is even or odd. As is customary, in our notation we shall generally suppress the inner product.

Denote by $C(V)$ the complex Clifford algebra of the real inner product space $(V, B)$; we may regard $C(V)$ either as the complexification of the real Clifford algebra of $(V, B)$ or as the Clifford algebra of the complexification $\left(V^{\mathbb{C}}, B^{\mathbb{C}}\right)$. Write $\gamma$ for the unique automorphism of $C(V)$ restricting to $V^{\mathrm{C}} \subset C(V)$ as minus the identity; this automorphism has period two and so determines a $Z_{2}$-grading

$$
C(V)=C_{0}(V) \oplus C_{1}(V)
$$

with $\gamma=I$ on the even Clifford algebra $C_{0}(V)$ and $\gamma=-I$ on the odd part $C_{1}(V)$.

Glasgow Math. J. 30 (1988) 263-270. 
Being a complexification, $C(V)$ comes equipped with a natural conjugation; this we shall indicate by an upper bar. Write $\alpha$ for the unique antiautomorphism of $C(V)$ restricting to $V^{\mathrm{C}} \subset C(V)$ as $-I$; the prescription

$$
u \in C(V) \Rightarrow u^{*}=\alpha(\bar{u})
$$

defines an antilinear involution on the complex algebra $C(V)$.

$C(V)$ is a full matrix algebra: there exists a complex vector space $S$ together with an algebra isomorphism

$$
\rho: C(V) \rightarrow \text { End } S
$$

we speak of $\rho$ as a spin representation of $C(V)$ with $S$ the corresponding space of spinors. Of course, any two spin representations of $C(V)$ are equivalent by an intertwining isomorphism which is unique up to scalar multiples.

The spin representation $\rho$ carries across the involution * on $C(V)$ to an involution * on End $S$; we claim that this determines a Hermitian form of some signature on $S$.

THEOREM. Every involution * on End $S$ is the adjoint operation determined by some Hermitian form ( ) on $S$, unique up to real scalar multiples.

Proof. Fix a Hermitian form $(\cdot, \cdot)$ on $S$, with adjoint operation ${ }^{\dagger}$ : if $\xi, \eta \in S$ and $T \in$ End $S$ then

$$
(T \xi, \eta)=\left(\xi, T^{\dagger} \eta\right)
$$

The composite of ${ }^{*}$ and ${ }^{\dagger}$ is an automorphism of End $S$, so there exists $\theta \in$ Aut $S$ such that

$$
T \in \text { End } S \Rightarrow T^{*}=\theta^{-1} T^{\dagger} \theta \text {. }
$$

Since involutions have period two, if $T \in$ End $S$ then

$$
T=T^{* *}=\left(\theta^{-1} \theta^{\dagger}\right) T\left(\theta^{-1} \theta^{\dagger}\right)^{-1}
$$

consequently, $\theta^{-1} \theta^{\dagger}$ is a scalar operator. Let $\theta^{\dagger}=\lambda \theta$ for some $\lambda \in \mathbb{C}$; then $\theta=\theta^{\dagger \dagger}=\bar{\lambda} \theta^{\dagger}$, whence $\lambda \bar{\lambda}=1$, so that if $\mu^{2}=\lambda$ then $(\mu \theta)^{\dagger}=\mu \theta$. We may therefore assume that $\theta^{\dagger}=\theta$. Define

$$
\langle\xi, \eta\rangle=(\theta \xi, \eta)
$$

for $\xi, \eta \in S$. Since $\theta^{\dagger}=\theta$ it follows that \langle\rangle is Hermitian, of the same signature as $\theta$ when $(\cdot, \cdot)$ is positive definite. Moreover, if $T \in$ End $S$ then

$$
\begin{aligned}
\langle T \xi, \eta\rangle & =(\theta T \xi, \eta) \\
& =\left(\left(T^{*}\right)^{\dagger} \theta \xi, \eta\right) \\
& =\left(\theta \xi, T^{*} \eta\right) \\
& =\left\langle\xi, T^{*} \eta\right\rangle
\end{aligned}
$$

whenever $\xi, \eta \in S$; thus, ${ }^{*}$ is the adjoint operation determined by \langle\rangle . It is evident that ${ }^{*}$ determines \langle\rangle to within scalar multiples. 
The space of spinors $S$ thus carries a canonical Hermitian form \langle\rangle such that the spin representation $\rho$ satisfies

$$
\langle\rho(u) \xi, \eta\rangle=\left\langle\xi, \rho\left(u^{*}\right) \eta\right\rangle
$$

whenever $u \in C(V)$ and $\xi, \eta \in S$.

The spin representation of the even Clifford algebra $C_{0}(V)$ splits as the direct sum of two irreducibles, called the half-spin representations on the spaces of half-spinors; we recall how this comes about. The anticentre of $C(V)$ is the set of all $a \in C(V)$ satisfying

$$
u \in C(V) \Rightarrow a u=\gamma(u) a .
$$

In fact, the anticentre of $C(V)$ is a complex line in $C_{0}(V)$ and contains an element $\omega$ such that $\omega^{2}=1$. The half-spin spaces $S_{+}$and $S_{-}$are the eigenspaces of $\rho(\omega)$ in $S$ corresponding to the eigenvalues +1 and -1 , so that $S=S_{+} \oplus S_{-}$. Note that since $\omega$ lies in the anticentre of $C(V)$, if $u_{0} \in C_{0}(V)$ then $\rho\left(u_{0}\right)$ stabilizes $S_{+}$and $S_{-}$whilst if $u_{1} \in C_{1}(V)$ then $\rho\left(u_{1}\right)$ interchanges $S_{+}$and $S_{-}$.

Let $\left(v_{1}, \ldots, v_{2 m}\right)$ be an orthonormal basis for $V$ with

$$
\begin{aligned}
& B\left(v_{j}, v_{j}\right)=+1(1 \leq j \leq p), \\
& B\left(v_{j}, v_{j}\right)=-1(p<j \leq 2 m) .
\end{aligned}
$$

In view of the anticommutation relations

$$
x, y \in V \Rightarrow x y+y x=2 B(x, y) 1
$$

in $C(V)$, it is readily seen that the product $v_{1} \ldots v_{2 m}$ lies in the anticentre of $C(V)$ and satisfies $\left(v_{1} \ldots v_{2 m}\right)^{2}=(-1)^{m+q}$. Thus: when $m+q$ is even, we may take $\omega=v_{1} \ldots v_{2 m}$; when $m+q$ is odd, we may take $\omega=i v_{1} \ldots v_{2 m}$. Note that in any case we have $\omega^{*}=(-1)^{q} \omega$.

As a first step in understanding the interaction of the canonical Hermitian form with the half-spinors, we have the following result.

THEOREM. If $B$ is even, then $S_{+}$and $S_{-}$are mutually orthogonal; if $B$ is odd, then $S_{+}$ and $S_{-}$are self-orthogonal.

Proof. If $\xi \in S_{+}$and $\eta \in S_{-}$then

$$
\begin{aligned}
\langle\xi, \eta\rangle & =\langle\rho(\omega) \xi, \eta\rangle \\
& =\left\langle\xi, \rho\left(\omega^{*}\right) \eta\right\rangle \\
& =(-1)^{q+1}\langle\xi, \eta\rangle ;
\end{aligned}
$$

it follows that the half-spin spaces $S_{+}$and $S_{-}$are mutually orthogonal when $q$ is even. Similarly, if $\xi, \eta \in S_{+}$or $\xi, \eta \in S_{-}$then

$$
\langle\xi, \eta\rangle=(-1)^{q}\langle\xi, \eta\rangle
$$

so that $S_{+}$and $S_{-}$are self-orthogonal when $q$ is odd. 
As an immediate corollary, if $B$ is odd then \langle\rangle has zero signature.

Suppose now that $B$ is even. According to the theorem, \langle\rangle restricts to a non-singular Hermitian form on each space of half-spinors. We claim: if $B$ is indefinite then \langle\rangle has zero signature on each half-spin space; if $B$ is definite then \langle\rangle is definite on each half-spin space, with like or opposite signs according to whether $B$ is negative or positive. Justification follows.

If $v \in V$ satisfies $B(v, v)= \pm 1$ then

$$
v^{*} v=\alpha(\bar{v}) v=-v^{2}=-B(v, v) 1=\mp 1 ;
$$

consequently, if $\xi, \eta \in S$ then

$$
\langle\rho(v) \xi, \rho(v) \eta\rangle=\left\langle\xi, \rho\left(v^{*} v\right) \eta\right\rangle=\mp\langle\xi, \eta\rangle ;
$$

moreover, $\rho(v)$ interchanges the half-spin spaces $S_{+}$and $S_{-}$.

THEOREM. If $B$ is even and indefinite then \langle\rangle has zero signature on each half-spin space.

Proof. From the above observation it is clear that the signatures of \langle\rangle on $S_{+}$and $S_{-}$ are both equal and opposite, and therefore zero.

More work is required when $B$ is definite. Our observation prior to the preceding theorem certainly implies that if $B$ is negative then \langle\rangle has the same signature on $S_{+}$and $S_{-}$whilst if $B$ is positive then the signatures of \langle\rangle on $S_{+}$and $S_{-}$are opposite. In order to establish that \langle\rangle is definite on $S_{+}$and $S_{-}$in either case, we digress to discuss the behaviour of spinors under orthogonal decompositions of $V$.

Suppose that $V$ is decomposed as the orthogonal sum $X \oplus Y$ of even-dimensional vector spaces. Let $S_{X}$ and $S_{Y}$ be spaces of spinors for $X$ and $Y$; write $S=S_{X} \otimes S_{Y}$. Fix $\omega$ in the anticentre of $C(X)$ with $\omega^{2}=1$ and define

$$
\rho: V \rightarrow \text { End } S
$$

by the prescription

$$
\rho(x \oplus y)=\rho(x) \otimes 1+\rho(\omega) \otimes \rho(y)
$$

for $x \in X$ and $y \in Y$. We claim that $\rho$ is a Clifford map: indeed, if $x \in X$ and $y \in Y$ then

$$
\rho(x \oplus y)^{2}=B(x \oplus y, x \oplus y) 1
$$

since $\omega^{2}=1$ and $\omega$ anticommutes with $x$. Simplicity of $C(V)$ and a dimensional check guarantee that the induced algebra map

$$
\rho: C(V) \rightarrow \text { End } S
$$

is actually an isomorphism. In this way, $S=S_{X} \otimes S_{Y}$ is realized as a space of spinors for $X \oplus Y$. The spaces of half-spinors in this realization are precisely

$$
\begin{aligned}
& S_{+}=\left(S_{X}\right)_{+} \otimes\left(S_{Y}\right)_{+}+\left(S_{X}\right)_{-} \otimes\left(S_{Y}\right)_{-}, \\
& S_{-}=\left(S_{X}\right)_{+} \otimes\left(S_{Y}\right)_{-}+\left(S_{X}\right)_{-} \otimes\left(S_{Y}\right)_{+} .
\end{aligned}
$$


Suppose further that $\omega^{*}=\omega$; this will be so iff $B$ is even on $X$. Let \langle\rangle$_{X}$ and \langle\rangle$_{Y}$ be canonical Hermitian forms on $S_{X}$ and $S_{Y}$. A Hermitian form \langle\rangle is defined on $S$ by the prescription

$$
\left\langle\xi \otimes \eta, \xi^{\prime} \otimes \eta^{\prime}\right\rangle=\left\langle\xi, \xi^{\prime}\right\rangle_{X}\left\langle\eta, \eta^{\prime}\right\rangle_{Y}
$$

for $\xi, \xi^{\prime} \in S_{X}$ and $\eta, \eta^{\prime} \in S_{Y}$. If $x \in X$ and $y \in Y$ then

$$
\begin{aligned}
& \left\langle\rho(x \oplus y)(\xi \otimes \eta), \xi^{\prime} \otimes \eta^{\prime}\right\rangle \\
& \quad=\left\langle\rho(x) \xi \otimes \eta+\rho(\omega) \xi \otimes \rho(y) \eta, \xi^{\prime} \otimes \eta^{\prime}\right\rangle \\
& \quad=\left\langle\rho(x) \xi, \xi^{\prime}\right\rangle_{X}\left\langle\eta, \eta^{\prime}\right\rangle_{Y}+\left\langle\rho(\omega) \xi, \xi^{\prime}\right\rangle_{X}\left\langle\rho(y) \eta, \eta^{\prime}\right\rangle_{Y} \\
& \quad=\left\langle\xi, \rho(-x) \xi^{\prime}\right\rangle_{X}\left\langle\eta, \eta^{\prime}\right\rangle_{Y}+\left\langle\xi, \rho(\omega) \xi^{\prime}\right\rangle_{X}\left\langle\eta, \rho(-y) \eta^{\prime}\right\rangle_{Y} \\
& \quad=-\left\langle\xi \otimes \eta, \rho(x) \xi^{\prime} \otimes \eta^{\prime}+\rho(\omega) \xi^{\prime} \otimes \rho(y) \eta^{\prime}\right\rangle \\
& \quad=-\left\langle\xi \otimes \eta, \rho(x \oplus y)\left(\xi^{\prime} \otimes \eta^{\prime}\right)\right\rangle
\end{aligned}
$$

whenever $\xi, \xi^{\prime} \in S_{X}$ and $\eta, \eta^{\prime} \in S_{Y}$. It follows that \langle\rangle is a canonical Hermitian form on $S$.

We are now essentially in a position to prove the following result.

THEOREM. If $B$ is negative definite on $V$ then \langle\rangle is definite on $S$. If $B$ is positive definite on $V$ then \langle\rangle is definite of opposite signs on $S_{+}$and $S_{-}$.

Proof. Induction on $m=\frac{1}{2} \operatorname{dim} V$. The case $m=1$ is presented explicitly as an example in the next section. In general let $V=X \oplus Y$ be an orthogonal decomposition into even-dimensional subspaces. We have seen that \langle\rangle is determined on $S=S_{X} \otimes S_{Y}$ as the product of \langle\rangle$_{X}$ and \langle\rangle$_{Y}$; we have also identified $S_{ \pm}$in terms of $\left(S_{X}\right)_{ \pm}$and $\left(S_{Y}\right)_{ \pm}$. From these observations and the mutual orthogonality of the half-spin spaces, it follows that if \langle\rangle$_{X}$ and \langle\rangle$_{Y}$ are definite on their respective half-spin spaces then so is \langle\rangle . It has already been established that the signs of \langle\rangle on $S_{+}$and $S_{-}$are equal or opposite according to whether $B$ is negative or positive.

We close this section with the following remarks. If $B$ is negative definite then $C(V)$ is a $C^{*}$ algebra in a natural manner. If $B$ is positive definite then $C_{0}(V)$ is a $C^{*}$ algebra but $C(V)$ is not: $u^{*} u$ has negative spectrum when $u \in C_{1}(V)$. If $B$ is indefinite then $C_{0}(V)$ is not a $C^{*}$ algebra: it contains a nonzero $u$ such that $u^{*} u=0$.

2. Some simple examples. Let $V$ be two-dimensional and let $B$ be definite. We shall prove that the canonical Hermitian form \langle\rangle is definite on $S_{+}$. In order to do this, it is enough to prove that $\langle\xi, \xi\rangle$ and $\langle\eta, \eta\rangle$ have the same sign whenever $\xi, \eta \in S_{+}$. Let $\eta \otimes \xi$ be the rank one operator defined on $S$ by the prescription

$$
\zeta \in S \Rightarrow(\eta \otimes \xi) \zeta=\langle\xi, \xi\rangle \eta
$$

and let $u \in C(V)$ correspond to $\eta \otimes \bar{\xi}$ under the spin representation $\rho$; since half-spin spaces are mutually orthogonal, $\eta \otimes \vec{\xi}$ stabilizes both $S_{+}$and $S_{-}$so that $u$ actually lies in 
$C_{0}(V)$. Note that

$$
(\eta \otimes \bar{\xi})^{*}(\eta \otimes \bar{\xi})=\langle\eta, \eta\rangle \xi \otimes \xi
$$

consequently, the spectrum of $u^{*} u$ is given by

$$
\sigma\left(u^{*} u\right)=\{\langle\xi, \xi\rangle\langle\eta, \eta\rangle\} .
$$

Thus, it suffices to show that the spectrum of $u^{*} u$ is positive whenever $u \in C_{0}(V)$.

Take an orthonormal basis $\{x, y\}$ for $V$; thus, $x$ and $y$ are mutually orthogonal and $B(x, x)=B(y, y)= \pm 1$. The elements 1 and $x y$ provide a basis for $C_{0}(V)$. For our purposes, a more convenient basis is given by

$$
\left\{\pi_{+}=\frac{1}{2}(1+\omega), \pi_{-}=\frac{1}{2}(1-\omega)\right\}
$$

where $\omega=i x y$; note that $\rho\left(\pi_{+}\right)$and $\rho\left(\pi_{-}\right)$project $S$ onto the half-spin spaces $S_{+}$and $S_{-}$. In fact, $\pi_{+}$and $\pi_{-}$are complementary mutually orthogonal selfadjoint idempotents:

as is readily verified. Let

$$
\begin{gathered}
\pi_{+}^{2}=\pi_{+}=\pi_{+}^{*}, \quad \pi_{-}^{2}=\pi_{-}=\pi_{-}^{*}, \\
\pi_{+} \pi_{-}=\pi_{-} \pi_{+}=0, \\
\pi_{+}+\pi_{-}=1,
\end{gathered}
$$

$$
u=a \pi_{+}+b \pi_{-}
$$

be any element of $C_{0}(V)$. From the above properties of $\pi_{+}$and $\pi_{-}$we deduce

$$
u^{*} u=|a|^{2} \pi_{+}+|b|^{2} \pi_{-}
$$

consequently,

$$
\sigma\left(u^{*} u\right)=\left\{|a|^{2},|b|^{2}\right\} .
$$

We have now justified our claim that if $B$ is definite then \langle\rangle is definite on $S_{+}$.

For completeness we describe $\sigma\left(u^{*} u\right)$ for $u \in C_{1}(V)$. As basis for $C_{1}(V)$ we take

$$
\left\{v_{+}=\frac{1}{2 i}(x+i y), v_{-}=\frac{1}{2 i}(x-i y)\right\} \text {. }
$$

Let $\delta= \pm 1$ be the common value of $B(x, x)$ and $B(y, y)$. If $\delta=-1$ then $v_{+} v_{-}=\pi_{+}$and $v_{-} v_{+}=\pi_{-}$whilst if $\delta=+1$ then $v_{+} v_{-}=-\pi_{-}$and $v_{-} v_{+}=-\pi_{+}$; in either case we have

$$
\begin{aligned}
& v_{+}^{2}=v_{-}^{2}=0, \\
& v_{+}^{*}=v_{-}, \quad v_{-}^{*}=v_{+} .
\end{aligned}
$$

As an arbitrary element of $C_{1}(V)$ consider

$$
u=a v_{+}+b v_{-}
$$

we have

$$
\begin{aligned}
& \delta=-1 \Rightarrow u^{*} u=+\left(|b|^{2} \pi_{+}+|a|^{2} \pi_{-}\right), \\
& \delta=+1 \Rightarrow u^{*} u=-\left(|a|^{2} \pi_{+}+|b|^{2} \pi_{-}\right),
\end{aligned}
$$


and therefore

$$
\sigma\left(u^{*} u\right)=\left\{-\delta|a|^{2},-\delta|b|^{2}\right\} .
$$

Thus: if $u \in C_{1}(V)$ then the spectrum of $u^{*} u$ is positive when $B$ is negative definite and negative when $B$ is positive definite.

When $B$ is indefinite, so that \langle\rangle vanishes on each space of half-spinors, it can be shown that if $u \in C_{0}(V)$ then $\sigma\left(u^{*} u\right)$ consists of a pair of complex conjugates; in this case, it is convenient to take $\frac{1}{2}(1+x y)$ and $\frac{1}{2}(1-x y)$ as basis vectors for $C_{0}(V)$.

As a further example we consider Minkowski space: a four-dimensional real vector space $V$ with an inner product $B$ of type $(1,3)$. From our general theory, the full four-dimensional complex vector space $S$ of spinors for $(V, B)$ is naturally provided with a Hermitian form \langle\rangle ; since $B$ is odd, \langle\rangle vanishes on each space of half-spinors. When taken with this canonical Hermitian form \langle\rangle$, S$ becomes what is known as twistor space.

It is perhaps worthwhile to present an explicit construction of Minkowski space, along with the spin representation and associated Hermitian form; this we proceed to do.

Let $S_{+}$be a two-dimensional complex vector space equipped with a complex symplectic form $\varepsilon$. The conjugate space to $S_{+}$will be denoted $S_{-}=\bar{S}_{+}$; the identity map from $S_{+}$to $S_{-}$is antilinear and will be denoted by an upper bar. An antilinear automorphism $\tau$ of $S_{+} \otimes S_{-}$is determined by the requirement

$$
\tau(p \otimes \bar{q})=q \otimes \bar{p}
$$

for $p, q \in S_{+}$. We take $V$ to be the four-dimensional real vector space of points fixed under $\tau$, so that $S_{+} \otimes S_{-}$is naturally the complexification of $V$. A symmetric bilinear map $B$ is defined on $S_{+} \otimes S_{-}$by requiring

$$
B(p \otimes \bar{q}, r \otimes \bar{s})=\varepsilon(p, r) \overline{\varepsilon(q, s)}
$$

to hold for all $p, q, r, s \in S_{+}$. The restriction of $B$ to $V$ is a real inner product of type $(1,3)$ : indeed, if $\{e, f\}$ is a basis for $S_{+}$with $\varepsilon(e, f)=\frac{1}{\sqrt{2}}$ then the vectors

$$
\begin{aligned}
& t=e \otimes \bar{e}+f \otimes \bar{f} \\
& x=e \otimes \bar{e}-f \otimes \bar{f} \\
& y=e \otimes \bar{f}+f \otimes \bar{e} \\
& z=i\{e \otimes \bar{f}-f \otimes \bar{e}\}
\end{aligned}
$$

are mutually orthogonal in $V$ and satisfy

$$
-B(t, t)=B(x, x)=B(y, y)=B(z, z)=-1 \text {. }
$$

$(V, B)$ is our model of Minkowski space.

The direct sum $S=S_{+} \oplus S_{-}$is a canonical space of spinors for Minkowski space. 
Define

$$
\frac{1}{\sqrt{2}} \rho(p \otimes \bar{q})(a \oplus \bar{b})=\overline{\varepsilon(b, q)} p \oplus \varepsilon(p, a) \bar{q}
$$

for $p, q, a, b \in S_{+}$. It is routine to verify that if $p, q, r, s \in S_{+}$then

$$
\rho(p \otimes \bar{q}) \rho(r \otimes \bar{s})+\rho(r \otimes \bar{s}) \rho(p \otimes \bar{q})=2 B(p \otimes \bar{q}, r \otimes \bar{s}) I ;
$$

consequently,

$$
\rho: S_{+} \otimes S_{-} \rightarrow \operatorname{End}\left(S_{+} \oplus S_{-}\right)
$$

is a Clifford map. The induced algebra map

$$
\rho: C(V) \rightarrow \text { End } S
$$

is actually an isomorphism, for any number of reasons. This exhibits $S$ as a space of spinors for Minkowski space. Observe that $S_{+}$and $S_{-}$are the corresponding spaces of half-spinors, as our notation suggests.

In searching for a Hermitian form \langle\rangle on $S=S_{+} \oplus S_{-}$satisfying

$$
\langle\rho(u) \xi, \eta\rangle=\left\langle\xi, \rho\left(u^{*}\right) \eta\right\rangle
$$

whenever $u \in C(V)$ and $\xi, \eta \in S$, we may be guided by its abstract properties. The formula

$$
\langle a \oplus \bar{b}, c \oplus \bar{d}\rangle=i\{\varepsilon(a, d)-\overline{\varepsilon(c, b)}\},
$$

for $a, b, c, d \in S_{+}$, determines such a form: if $p, q \in S_{+}$then $(p \otimes \bar{q})^{*}=-q \otimes \bar{p}$ and a direct calculation verifies

$$
\langle\rho(p \otimes \bar{q})(a \oplus \bar{b}), c \oplus \bar{d}\rangle+\langle a \oplus \bar{b}, \rho(q \otimes \bar{p})(c \oplus \bar{d})\rangle=0
$$

whenever $a, b, c, d \in S_{+}$.

In this treatment of Minkowski space, responsibility for the explicit formula giving the spin representation $\rho$ of $S_{+} \otimes S_{-}$on $S_{+} \oplus S_{-}$is shared with Roger Plymen.

\section{REFERENCES} 3-38.

1. M. F. Atiyah, R. Bott and A. Shapiro, Clifford modules, Topology 3 (1964), Supplement,

2. N. Bourbaki, Algèbre, Chapitre 9 (Hermann, 1959).

3. C. Chevalley, The algebraic theory of spinors (Columbia University Press, 1954). 1981).

4. R. Deheuvels, Formes quadratiques et groupes classiques (Presses Universitaires de France,

5. W. Greub, Multilinear algebra, second edition (Springer-Verlag Universitext, 1978).

SCHOOL OF MATHEMATICS

Trinity College

DUBLIN 2

IRELAND
Present address:

Department of Mathematics

UNIVERSITY OF FLORIDA

GAINESVILLE

FLORIDA 32611

U.S.A. 